\title{
J1026+2542: proper motion in a blazar jet at $z=5.27$
}

\author{
Sándor Frey ${ }^{* \dagger}$ \\ FÖMI Satellite Geodetic Observatory, Hungary \\ E-mail: frey.sandor@fomi.hu
}

\section{Zsolt Paragi}

Joint Institute for VLBI in Europe, The Netherlands

\section{Judit O. Fogasy}

Onsala Space Observatory, Chalmers University of Technology, Sweden

\section{Leonid I. Gurvits}

Joint Institute for VLBI in Europe, The Netherlands

Dept. of Astrodynamics and Space Missions, Delft Univeristy of Technology, The Netherlands

\begin{abstract}
The radio-loud AGN J1026+2542 has recently been classified as the second most distant blazar, based on its broad-band spectral energy distribution and X-ray spectrum. The source with a prominent one-sided jet extending to at least $\sim 20$ mas was earlier observed with the VLBA at 5 GHz in January 2006. With our new EVN observation (May 2013), we detected the displacement and directly measured the apparent proper motion of the jet components, for the first time at such a high redshift. The 1.6-GHz EVN observation provided additional information on the extent and the spectral properties of the jet. The VLBI results are consistent with the picture in which J1026+2542 has its jet oriented close to the line of sight, with significant Doppler boosting and a large bulk Lorentz factor.
\end{abstract}

12th European VLBI Network Symposium and Users Meeting,

7-10 October 2014

Cagliari, Italy

\section{* Speaker.}

$\dagger$ The EVN is a joint facility of European, Chinese, South African, and other radio astronomy institutes funded by their national research councils. S.F. and Z.P. thank the Hungarian Scientific Research Fund (OTKA K104539) for support. The research leading to these results has received funding from the European Commission 7th Framework Programme (FP/2007-2013) under grant agreement No. 283393 (RadioNet3). 


\section{Introduction}

Blazars are radio-emitting active galactic nuclei (AGN) prominent in other wavebands as well. The number of known blazars is steadily increasing, owing to the sky surveys at radio, infrared, optical, X-ray, and $\gamma$-ray bands. However, confirmed blazars at extremely high redshifts $(z>5)$ are still rare: there are only three of them known at present. The second most distant one is $\mathrm{J} 1026+2542$ at $z=5.266[6,7]$.

The radio emission from the blazar jets can be imaged with the highest angular resolution using very long baseline interferometry (VLBI). Typical observed features are compact flat-spectrum "cores", one-sided jets, high Doppler-boosted brightness temperature, and often apparent superluminal motion of jet components. These can be reconciled with a synchrotron jet model where the relativistic outflow of plasma is inclined at a very small angle to the line of sight. The source $\mathrm{J} 1026+2542$ is unique at such a high redshift because it shows a prominent jet structure with multiple components extending out to tens of milli-arcseconds (mas). It is also unique because its first VLBI imaging observations at $5 \mathrm{GHz}$ date back to 2006 [4]. The rich jet structure and the first-epoch reference measurement prompted us to look for possible changes in the radio jet of J1026+2542, by means of repeated VLBI imaging at the same observing frequency.

VLBI monitoring observations are a useful direct tool to study kinematic properties of AGN jets. By measuring the apparent component speeds and estimating the Doppler-boosting factor in an independent way, it is possible to infer the Lorentz factor of the plasma flow and the jet orientation with respect to the line of sight. Apart from studying the jet physics, the measured apparent proper motions in a large sample of jet components over a broad range of redshifts can also serve as a test for cosmological models (e.g. [5]). With just a two-epoch estimate of jet component proper motions, one cannot compete with extensive multi-epoch AGN surveys, but any new kinematic data for sources at extremely high redshifts extend the apparent proper motion-redshift relation to a yet unexplored terrain.

\section{EVN observations and results}

We observed J1026+2542 with the EVN at $5 \mathrm{GHz}$ on 2013 May 28, and at $1.7 \mathrm{GHz}$ on 2013 June 4 (experiment EF024). The resulting VLBI images are shown in Fig. 1. Due to its optically thin steep-spectrum emission, the diffuse end of the jet can be traced out to $\sim 50$ mas in the 1.7- $\mathrm{GHz}$ EVN image, corresponding to $\sim 300 \mathrm{pc}$ projected linear size (assuming a cosmological model with $H_{0}=70 \mathrm{~km} \mathrm{~s}^{-1} \mathrm{Mpc}^{-1}, \Omega_{\mathrm{m}}=0.3$, and $\left.\Omega_{\Lambda}=0.7\right)$. At $5 \mathrm{GHz}$, the second-epoch imaging gives a time baseline of $7.33 \mathrm{yr}$ with respect to the earlier Very Long Baseline Array (VLBA) observations [4]. In the rest frame of the source, due to the time dilation caused by the expansion of the Universe, this corresponds to a period shorter by a factor of $(1+z)$, i.e. $1.17 \mathrm{yr}$. Details of the observations and data analysis are given elsewhere [2].

Based on fitting Gaussian brightness distribution model components to the interferometric visibility data at both epochs (2006 and 2013), we detected structural changes in the jet and estimated significant apparent proper motions (0.09-0.11 mas $\left.\mathrm{yr}^{-1}\right)$ for three components. These correspond to superluminal apparent transverse velocities of $\sim 11-14 c$. Such proper motion estimates were 

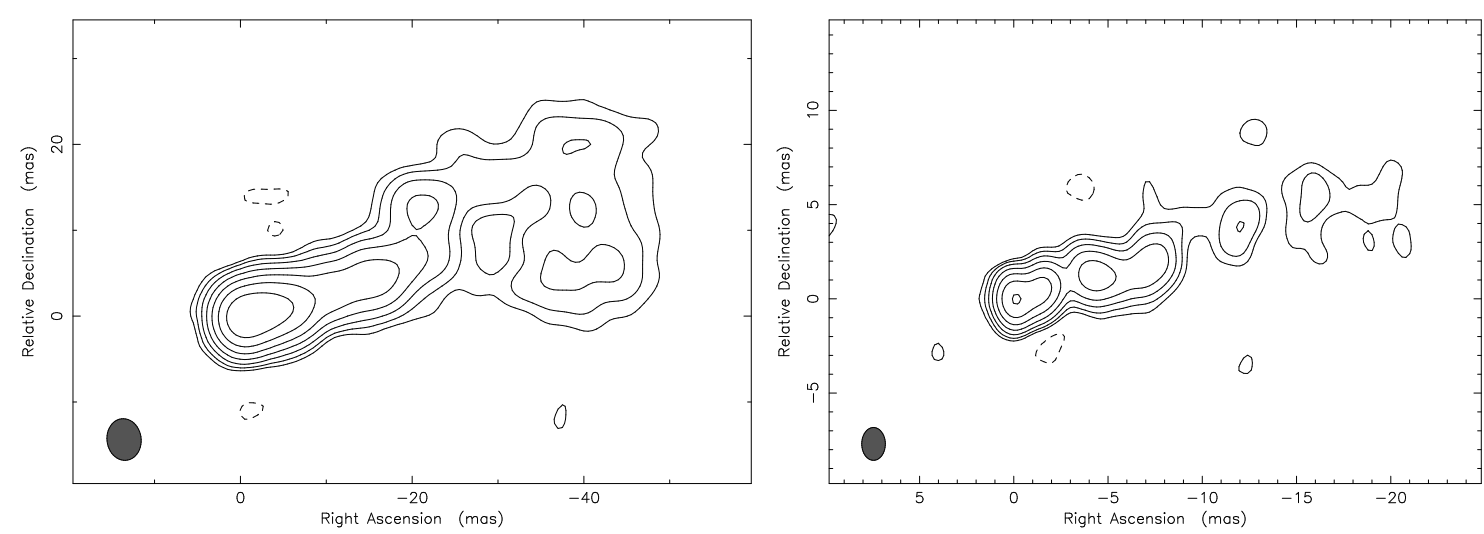

Figure 1: Naturally-weighted 1.7-GHz (left) and $5 \mathrm{GHz}$ (right) EVN images of J1026+2542. In the 1.7-GHz image, the lowest contours are at $\pm 0.45 \mathrm{mJy}_{\text {beam }}{ }^{-1}$, the peak brightness is $57.4 \mathrm{mJy}$ beam $^{-1}$, the Gaussian restoring beam is 4.87 mas $\times 3.98$ mas with major axis position angle $\mathrm{PA}=7.2^{\circ}$. In the $5-\mathrm{GHz}$ image, the lowest contours are at $\pm 0.32 \mathrm{mJy}^{\circ}$ beam $^{-1}$, the peak brightness is $21.7 \mathrm{mJy}_{\text {beam }}^{-1}$, the restoring beam is 1.74 mas $\times 1.25$ mas with $\mathrm{PA}=0.1^{\circ}$. In both images, positive contour levels increase by a factor of 2 . We show the coordinates relative to the phase centre.

made for the first time for any AGN at $z>5$. The moderate component motions are fully consistent with what is expected in a relativistic cosmological model.

The new EVN data allowed us to place a more stringent constraint on the size of the compact VLBI core. The lower limit to the core brightness temperature is $\sim 2.3 \times 10^{12} \mathrm{~K}$, the two-point spectral index in the core region is $\alpha=-0.35$ (defined as $S \propto v^{\alpha}$, where $S$ is the flux density and $v$ is the observing frequency). The flux densities in our VLBI components (180.4 mJy at $1.7 \mathrm{GHz}$ and $79.2 \mathrm{mJy}$ at $5 \mathrm{GHz}$ ) are $\sim 20-40 \%$ smaller than the total flux density values from the literature, most likely due to the presence of jet emission on angular scales of $\sim 100$ mas. This emission is resolved out by the VLBI observations. The overall radio spectrum of $\mathrm{J} 1026+2542$ follows a power law with spectral index $\alpha_{\text {total }}=-0.4$, in the observed frequency range of $151 \mathrm{MHz}-43 \mathrm{GHz}$ ([1] and references therein). Higher-frequency measurements suggest long-term variability and a steepening spectrum ( -0.7 spectral index) with increasing frequency [7].

The parameters derived for the inner radio jet from the EVN data are consistent with an independent estimate of the Lorentz factor $(\Gamma=13)$ and the viewing angle $\left(\theta=3^{\circ}\right)$ from high-energy measurements and the spectral energy distribution [7].

It is now right time to begin revisiting with VLBI imaging observations a larger sample of high-redshift $(z>3)$ radio quasars that show prominent jet structures in their first-epoch images, to estimate their apparent jet proper motions. The accumulating new observational data will be an essential supplement to the existing data bases for studying VLBI structures of inner AGN jets as cosmological probes [3].

\section{References}

[1] S. Frey et al., On the Doppler boosting in the compact radio jet of the distant blazar J1026+2542 at $z=5.3$, MNRAS 4311314 (2013) [arXiv:1302.2209] 
[2] S. Frey et al., The first estimate of radio jet proper motion at $z>5$, MNRAS 4462921 (2015) [arXiv:1410.8101]

[3] L.I. Gurvits, S. Frey, Z. Paragi, Jets in AGN at extremely high redshifts, in Proceedings of the IAU Symposium 313, Extragalactic jets from every angle, F. Massaro, C.C. Cheung, E. Lopez, A. Siemiginowska (eds.), Cambridge University Press, in press (2015) [arXiv:1410 . 8164]

[4] J.F. Helmboldt et al., The VLBA Imaging and Polarimetry Survey at 5 GHz, ApJ 658203 (2007) [arXiv:astro-ph/0611459]

[5] K.I. Kellermann et al., Kinematics of quasars and AGN, New Astron. Rev. 43757 (1999)

[6] T. Sbarrato et al., SDSS J102623.61+254259.5: the second most distant blazar at $z=5.3$, MNRAS 426 L91 (2012) [arXiv:1208.3467]

[7] T. Sbarrato et al., NuSTAR Detection of the Blazar B2 1023+25 at Redshift 5.3, ApJ 777147 (2013) [arXiv:1309.3280] 